\title{
INFLUENCE OF A ROOFING SYSTEM ON THE STATIC STABILITY OF RETICULATED SHELLS
}

\author{
Wen-Liang Li $^{1,2}$, Xu-Dong Zhi ${ }^{1,2 *}$, Duo-Zhi Wang ${ }^{3}$, Feng Fan ${ }^{1,2}$ and Shi-Zhao Shen ${ }^{1,2}$ \\ ${ }^{1}$ Key Lab of Structure Dynamic Behaviour and Control of the Ministry of Education, Harbin Institute of Technology, Harbin, China \\ ${ }^{2}$ Key Lab of Smart Prevention and Mitigation of Civil Engineering Disasters of Ministry of Industry and Information Technology, Harbin Institute of Technology, Harbin, China \\ ${ }^{3}$ Key Laboratory of Earthquake Engineering and Engineering Vibration, Institute of Engineering Mechanics, China Earthquake Administration, Harbin, China \\ *(Corresponding author: E-mail: zhixudong@hit.edu.cn)
}

\section{A B S T R A C T}

According to the existing experimental results, the roofing systems considerably influence the bearing capacity and plastic development of reticulated shell substructure models; however, the mechanism of this influence of the roofing system on the static stability of reticulated shell structures remains unclear. In this paper, based on experimental studies, the finite element model of reticulated shell structures with different roofing systems are established, and the influence of various factors of the reticulated shell structures (rise span ratio, roof load, initial imperfection amplitude and mode) and roofing system (pillar and purlin joint) are analysed. Furthermore, the influence ranges of the roofing system on the static bearing capacity of the shells are recommended.
A R T I C LE H IS T O RY

$\begin{array}{ll}\text { Received: } & \text { 19 February } 2020 \\ \text { Revised: } & \text { 12 September } 2020 \\ \text { Accepted: } & \text { 18 September } 2020\end{array}$

\section{K E Y W O R D S}

Reticulated shells

Roofing systems;

Static load;

Influences of different factors:

\section{Introduction}

With the continuous growth in society, the demand for large-span space buildings for production and recreational purposes is increasing. Architects often employ single-layer reticulated shell structures, owing to their low weight and reasonable force bearing capacity. An example of classical engineering is the Spectacle Roof of the MGM GRAND in Macau, which is the world's largest single-layer reticulated shell structure, providing people with a comfortable and extensive leisure and entertainment space. In recent years, scholars have systematically performed the analysis and design of reticulated shell structures. Shen Shizhao and Chen Xi [1] examined the elastic stability of single-layer shells and proposed design methods for different kinds of single-layer shell structures. Fan Feng et al. [2-3] studied the elastic-plastic stability of reticulated shell structures and established a modified design method. Zhi Xudong [4-5] studied the dynamic response and failure mechanism of single-layer reticulated shell structures. M. Fong, Y.P. Liu and S.L. Chan [6] employed a second-order analysis method to study the buckling load and failure mode of a shell with semi-rigid joints and initial imperfections, and the method was verified experimentally. Kato et al. [7-8] and Ma Huihuan [9-10] analysed the static stability of reticulated shell structures with different joint rigidities. Si-Wei Liu [11] used curved tapered-three-hinge beam column elements (TTH) to simulate a tapered member with I-sections in dome-like structures, the method exhibited a high calculation efficiency and accuracy and could be used to analyse practical engineering structures with tapered members. Furthermore, Rui Bai and Si-Wei Liu [12] proposed an efficient method using non-prismatic elements to analyse the dynamic response of frame structures with tapered sections members. As an innovative approach, Y.P. Liu and S.L. Chan [13-14] applied the second-order direct analysis (SODA) method and TTH method to the design and construction of the MGM Spectacle Roof in Macau, the construction and offload processes of the Spectral Roof were monitored and compared with the analysis results of the SODA method, thereby ensuring the safety and accuracy of the construction process, the practicability of the analysis method was also verified in this manner. Xie Xiangyang [15] analysed the key design and analysis technologies of reticulated shells with prism joints in the construction of the MGM Spectacle Roof.

The aforementioned studies provided valuable guidance for the analysis of single-layer reticulated shell structures under static and seismic loads. However, the influence of a roofing system on the reticulated shell structures was not considered in most of the existing studies, and this simplification may induce deviations in the research findings of reticulated shell structures. In the current steel structure design codes [16-19], the influence of roofing systems on the bearing capacity of the main structure are not clearly specified, even though these elements are considered as the safety reserve of the structures, the influence characteristics of the roofing systems on reticulated shell structures has not been clarified. Several scholars attempted to examine the influence of the roofing systems on the static and seismic performances of long-span space structures. He Yongjun [20] analysed the influence of concrete roof panels on the static stability of grid structures and established a simplified finite element analysis method pertaining to the concrete panels in grid structures. Zhang Yigang [21] analysed the influence of metal-faced roofing panels on the frequency response of cylindrical reticulated shells, by performing a shaking table test. Maria Koliou [22-23] analysed the seismic performance of long-span structures with roof and wall panels and observed that the roof and wall panels could improve the seismic performance of the main structures. Zhi Xudong and Li Wenliang [24-26] conducted static loading experiments using single-layer reticulated shell substructure models, considering different types of roofing systems, as shown in Fig. 1, the static and dynamic characteristics of a reticulated shell with a roofing system were preliminarily analysed.

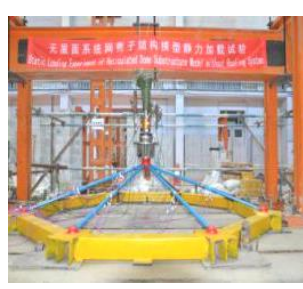

(a) Substructure model without roofing system

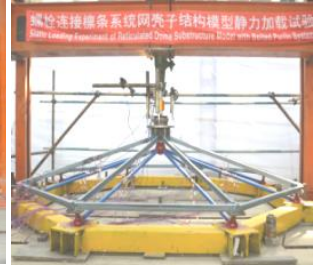

(b) Substructure model with only purlin system

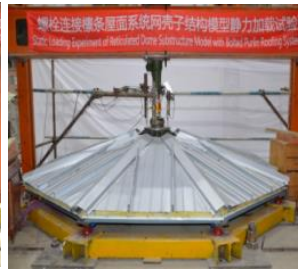

(c) Substructure model with complete roofing system
Fig. 1 Finite element model of the roofing system

According to the aforementioned studies, roofing systems notably influence the static and dynamic characteristics of long-span space structures. However, there are still some important issues to be addressed: (1) mechanism of the effect of roofing systems on the static stability of reticulated shells and (2) the characteristics of different roofing systems influences on different reticulated shell structures static bearing capacity need to be further studied, and the influence range should also be obtained.

\section{Finite element model of the reticulated shell structure with a roofing system}

The shell members and roofing system components in the structures are designed according to the relevant requirements in the current Chinese specifications [16,19]. All the roof panels in the finite element models (FEMs) are simulated according to the commonly implemented installation processes 
for roof panels in China, as shown in Fig. 2(a). The bottom panels in the reticulated shell are shallow profiled steel sheets fixed on purlins through self-tapping screws. Subsequently, the insulating layers are laid on the bottom panels and secondary purlins, as shown in Fig. 2(b). Finally, the profiled metal-faced panels are compacted onto the supports, which are fixed on the secondary purlins, as shown in Fig. 2(c). When using the current installation technologies for roofing systems, the insulation layer, faced panels and secondary purlins do not notably affect the static stability of the shells and are thus not considered in the following analysis.

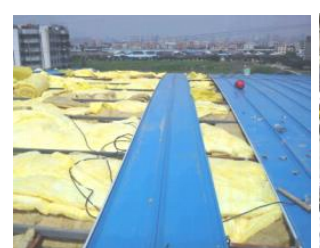

(a) Installation process of the roof panel in an actual engineering application

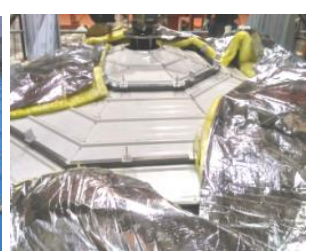

(b) Procedure of laying the insulating glass cotton

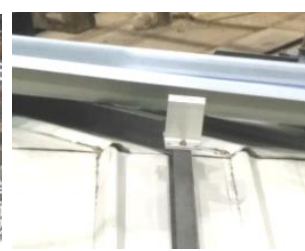

(c) Procedure of laying the faced panels
Fig. 2 Process of installing the roof panels

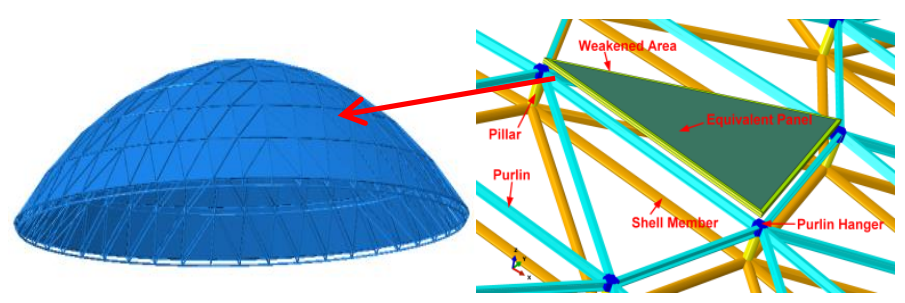

Fig 3 Finite element model of the roofing system

Fig. 3 shows that the FEM includes the purlin system, purlin hangers, equivalent orthotropic panel, weakened areas, pillars and reticulated shell. Specifically, the connections between the panel and purlins are simulated as the weakened areas, and the orthotropic panel is used to simulate the shallow profiled steel sheet for the roof panel. The mechanical properties are researched according to the experimental results and finite element analysis (FEA) according to Ref [24]. In practical engineering applications, bolted or welded connections are widely used in purlin joints, the mechanical properties of which are different from those of hinge or fixed joints. Xie Xiangyang [27] analysed semi-rigid joints with six degrees of freedom and applied such joints in steel tubular scaffolds with couplers. In general, the method of simulating semi-rigid joints is highly reliable, and which can be used to model the purlin joints for the shells with a roofing system, taking into account the rigidity and plastic development of the purlin joints. The constitutive models for the FEM are ideal elastic-plastic models, and the elastic modulus and yield strength of the shell members, purlin, and pillars are $206 \mathrm{GPa}$ and $235 \mathrm{MPa}$, respectively..

\section{The reticulated shell finite element analysis with roofing system}

According to the modelling method described in Section 2, the effects of the roofing system on the static stability of the reticulated shell structures are analysed.
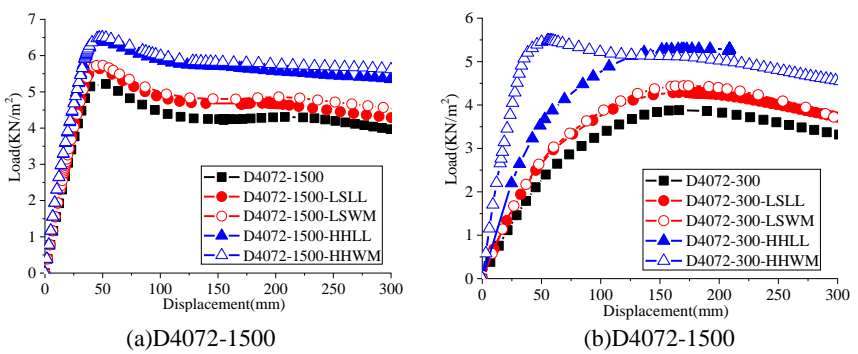

Fig. 4 Load-displacement curves of D4072

Fig. 4 shows that the bolted purlin system increases the static bearing capacity of the reticulated shell with the L/1500 initial imperfection by $8.27 \%$, the complete bolted purlin roofing system increases the static bearing capacity by $10.1 \%$. When the purlin joints are used as welded connections, the bearing capacity is increased by $22.1 \%$, and after the addition of the roof panels, the bearing capacity is increased by $24.8 \%$. Fig. 5 shows that because of the roofing system, the plastic shell members gradually develop towards the outer rings of the shell, the shell bearing capacity is respectively increased by $10.1 \%$ and $14.9 \%$ owing to the bolted purlin system and the complete bolted purlin roofing system for the shell with an L/300 initial imperfection. The influences of the welded purlin system on the static bearing capacity are more significant; specifically, this system increases the bearing capacity by $36 \%$, moreover, the complete welded purlin roofing systems increase the static bearing capacity by $41.4 \%$.

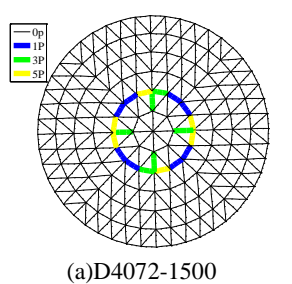

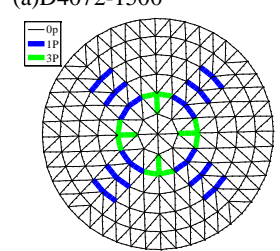

(d)D4072-1500-HHLL
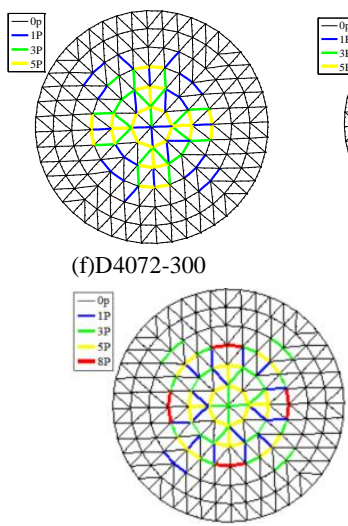

(i)D4072-300-HHLL

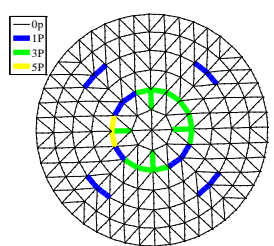

(b)D4072-1500-LSLL

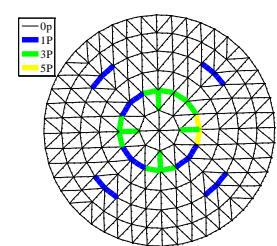

(c)D4072-1500-LSWM

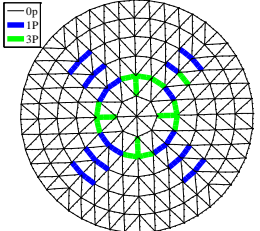

(e)D4072-1500-HHWM

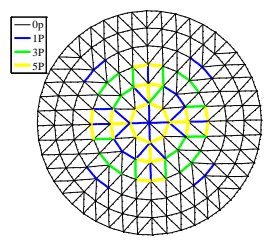

(g)4072-300-LSLL

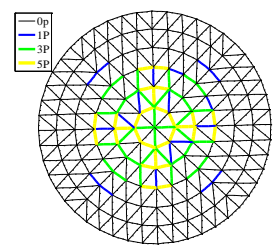

(h)D4072-300-LSWM

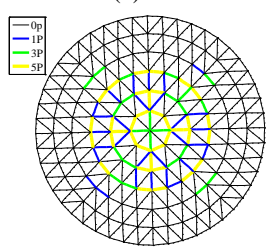

(j)D4072-300-HHWM

Fig. 5 Distribution of plastic members in D4072

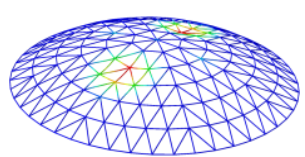

(a) $\mathrm{D} 4072-1500$

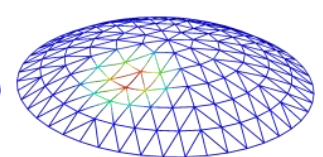

(b)D4072-1500-LSLL

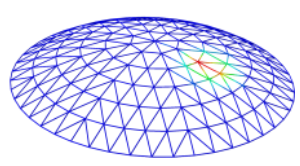

(c)D4072-1500-LSWM

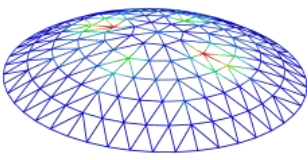

(d)D4072-1500-HHLL

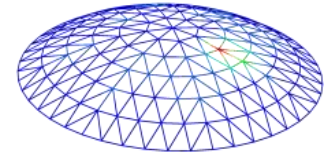

(e)D4072-1500-HHWM

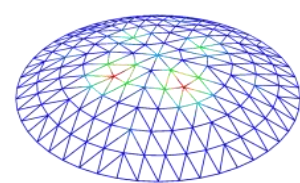

(f)D4072-300

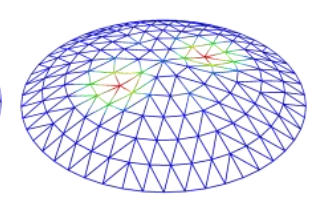

(g)4072-300-LSLL

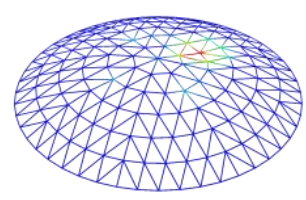

(h)D4072-300-LSWM

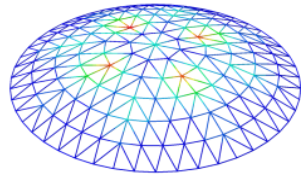

(i)D4072-300-HHLL

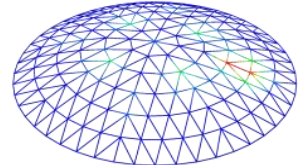

(j)D4072-300-HHWM

Fig. 6 Deformation in D4072

Fig. 6 indicates that roofing system changed the failure mode of the reticulated shell structure. The failure mode of the reticulated shell 
(D4072-1500) without a roofing system is symmetrical, and notable depressions develop continuously and collapse on the two vertices of the secondary ring around the centre vertex. After installing the roofing system, the reticulated shell structure exhibits a notable unilateral depression, and the local damage of the reticulated shell structure becomes more significant, as shown in Figs. 6(c) and 6(e). Moreover, Figs. 6(f) and. 6(j) show that the location of damage in the shell changes owing to the welded roofing system, and the local damage phenomena become more significant. The static bearing capacity of the single-layer spherical reticulated shell structure primarily depends on the bending and membrane rigidity around the vertices on the main rib rings of the reticulated shell structures. When the purlin system and roof panels are installed, all these members act as composite beams to bear an external load along with the shell members. In the scenario, the bending and membrane rigidity of the shell increase, and the bearing capacity of the reticulated shell structures with the roofing system increases significantly. In general, the static bearing capacity is closely related to many factors such as the initial imperfection amplitude and mode, axial load-displacement and bending moment rotation relationships of the purlin joint, and pillar height, the influence of these factors should be further analysed.

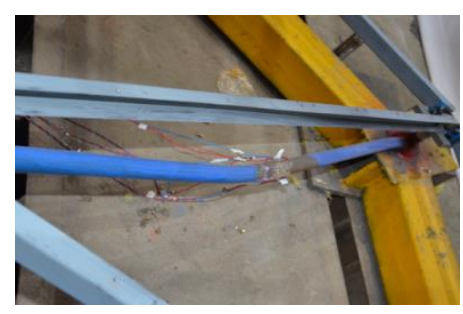

(a) Deformation of the purlin and shell members

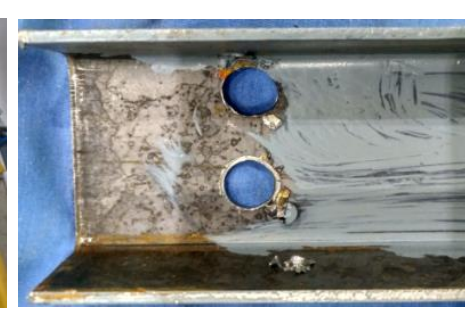

(b) Plastic deformation at the location of the bolted hole
Fig. 7 Deformation of the shell members and bolted purlin in the experimental models

In general, the effect of the bolted purlin roofing system on the static stability of a shell is not as significant as that of the welded roofing system, because premature yielding occurs at the locations of the bolted holes, while there is no plastic deformation occurs in the purlin members. The same phenomenon is also observed in the experimental studies, as shown in Fig. 7, even at the end of the experiments, the deformations at most of the purlins are not notable, while significant plastic deformation can be observed near the bolted hole of the bolted purlin connections. In the case of the bolted and welded purlin roofing systems, when D4072-1500 exhibits the highest static bearing capacity, $70.2 \%$ and $72.4 \%$ of the purlin joints yield in the axial restraint direction, respectively, and $12.7 \%$ and $2.2 \%$ of the purlin joints yield in the out-of-surface moment directions, respectively. In the bolted and welded roofing systems, when D4072-300 exhibits the highest bearing capacity, $44.6 \%$ and $56 \%$ of the purlin joints yield in the axial restraint direction, respectively, and $45 \%$ and $18.64 \%$ of the purlin joints yield in the out-of-surface moment directions, respectively. The torsional moment and the moment in the surface of the purlin joints are nearly still in elastic stages. The out-of-surface and axial constraints of the purlin joints are the main factors facilitating the increase in the shell bearing capacity owing to the roofing system.

\section{Influence of different factors on the static stability of reticulated shell structures with roofing systems}

The aforementioned analysis results show that the roofing system's influences on the shell's static stability are obvious, and further analysis should be carried out according to different influence factors in the reticulated shell and the roofing system, as shown in Table 1.

In this study, except when analysing the influence of the imperfection modes, the 1st order buckling mode of the reticulated shells is considered as the initial imperfection mode for the calculations.

\subsection{Influence of the rise span ratios}

The effect of the span on the static stability of the shells with roofing systems was analysed in Ref [24]. In this section, the influence of the roofing systems on the static stability of the shells, corresponding to rise span ratios of $1 / 3,1 / 5$ and $1 / 7$ are analysed.
Table 1

Parameter analysis
Rise span ratios

Initial imperfection amplitude

Initial imperfection modes

The purlin joint's rigidity

Height of the pillar (m)
$1 / 3,1 / 5,1 / 7$

$0, \mathrm{~L} / 1500, \mathrm{~L} / 300$

The 1st-order buckling mode, the consistent imperfection mode (1), the consistent imperfection mode

(2), the 1st-order frequency mode

$0.2,1.0,2.0$

$0.2,0.6,1.0$

$35 / 50,95 / 50,155 / 50$
Roof load $\left(\mathrm{kg} / \mathrm{m}^{2}\right)$

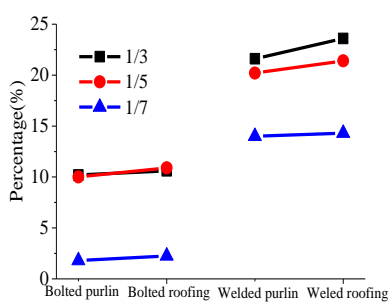

(a) L/300-imperfection

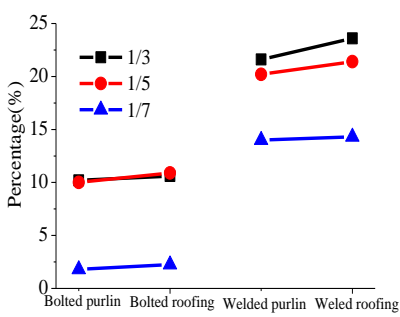

(b) L/1500-imperfection

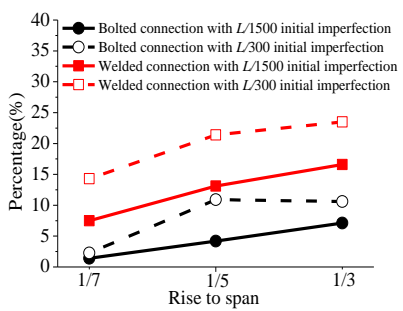

(c) Percentage improvement with the rise span

Fig. 8 Percentage improvement in the bearing capacity of the shells with different rise span ratios

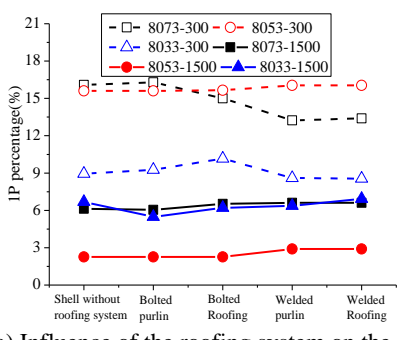

(a) Influence of the roofing system on the $1 \mathrm{P}$ percentages

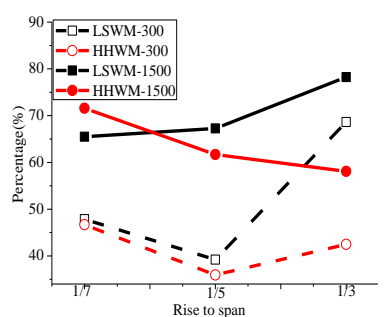

(b) Yield ratio of the purlin joint in the axial force direction

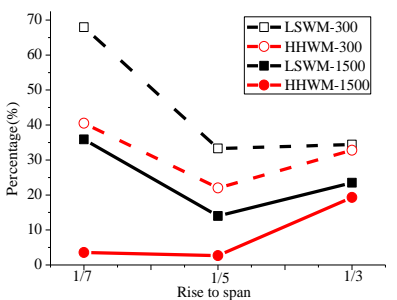

(c) Yield ratio of the purlin joint in the bending moment in the out-of-surface direction

Fig. 9 Influence of the roofing system on the plastic member and plastic purlin joint

percentage

The effects of the roofing system on the static stability of the shell are different under different rise span ratios. Fig. 8 shows that as the rise span ratios increase, the effect of the roofing system on the static bearing capacity becomes more notable. Fig. 9(a) shows that the roofing systems do not significantly influence the proportion of the plastic members in the reticulated shells under the three rise span ratios (changes within 3\%). No plastic deformation occurs in the purlin members and roof panels; however, a large number of axial constraints and out-of-surface bending moment constraints in 
the purlin joints exhibited yielding, as shown in Figs. 9 (b) and (c). As the rise span ratio increases in the shell with the L/1500 initial imperfection, the yield ratio of the axial constraints and out-of-surface moment constraints of the welded purlin joints decreases and increases, respectively. The yield ratio of the axial restraint for both kinds of purlin joints is significantly higher than that of the out-of-surface bending moment constraints, thereby indicating that the axial restraint of the purlin joint more notably influences the increase in the bearing capacity of a shell with a smaller initial imperfection (L/1500). The yield ratio of the out-of-surface bending moment constraint significantly increases in the case of the reticulated shell structures with an initial imperfection of L/300, and the out-of-surface moment constraints are more notably factors to influence the the static bearing capacity of a shell with a roofing system with a smaller rise span ratio (1/7).

\subsection{Influence of the initial geometric imperfections}

The initial geometric imperfections influence the static stability of the reticulated shell structures; therefore, the cases in which both the roofing systems and reticulated shells contain initial imperfections need further analysis. The amplitudes of the initial imperfection are L/1500, L/900 and $\mathrm{L} / 300$. The 1st-order buckling modes of the reticulated shells are used as the initial imperfection modes, and the influences of the roofing systems on the reticulated shells under three kinds of spans and rise span ratios are analysed.

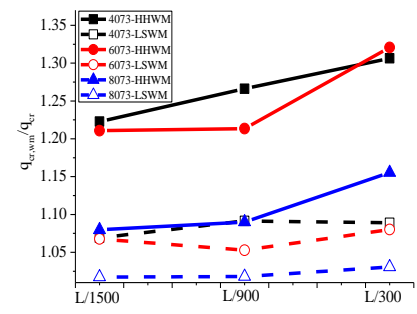

(a) Ratio of the bearing capacity of shells with different spans

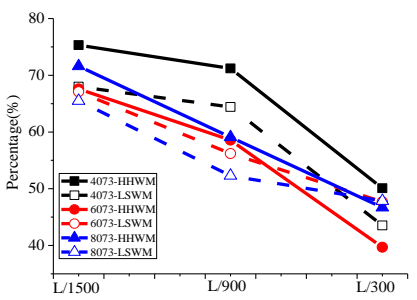

(c) Axial constraint yield ratio in shells with different spans

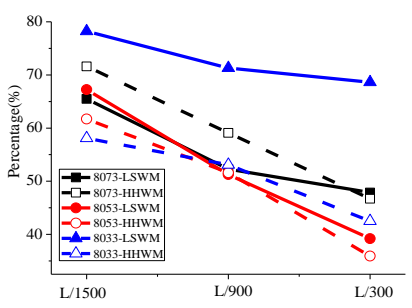

(e) Axial constraint yield ratio in shells with different rise spans

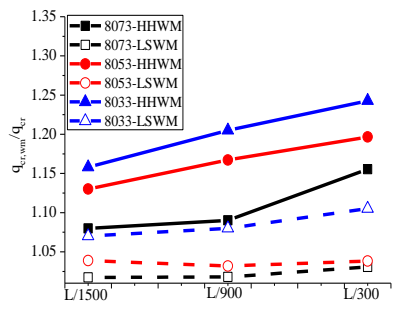

(b) Ratio of the bearing capacity of shells with different rise spans

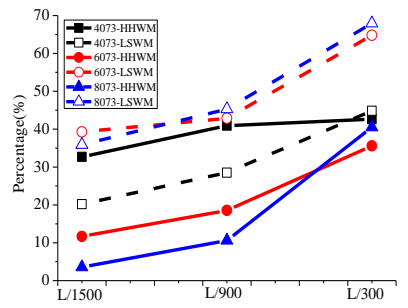

(d) Bending moment out-of-surface yield ratios in shells with different spans

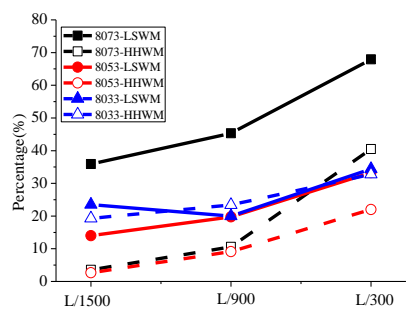

(f) Bending moment out-of-surface yield ratio in shells with different rise spans
Fig. 10 Influence of the amplitudes of the initial imperfections on the bearing capacity and purlin joint yield ratio of shells with a roofing system

According to the aforementioned analysis, the roofing system considerably influences the shells with different initial imperfection amplitudes, and the influence of the roofing system on the static bearing capacity tends to increase with an increase in these amplitudes, as shown in Figs. 10 (a) and (b). The increase in the static stability of the reticulated shells owing to the welded purlin roofing system is higher under a smaller span and larger imperfection amplitude. The increase in the static bearing capacity of the shell is not as notably influenced by the initial imperfection amplitudes in the case of the bolted purlin roofing system as that in the case of the welded purlin roofing system (the fluctuation amplitude is within 3\%).

Figs. 10(c)-(f) show that as the amplitude of the initial geometric imperfection increases, the yield proportion of the axial constraints and out-of-surface bending moment constraints in the purlin joints gradually decreases and increases, respectively. This phenomenon occurs because the mechanism of the membrane stress in the reticulated shell with a smaller initial imperfection is more significant, and more axial direction constraints act on the bearing external load as well as on the reticulated shell, owing to which, a larger number of axial direction constraints enter the plastic stage. Additionally, when the initial imperfection amplitude are larger, the reticulated shell exhibits a continuous depression around the locations of the initial imperfections, owing to which the shell stops effectively transmitting the membrane force earlier, and more out-of-surface bending moment constraints in the purlin joints yield around the concave position.
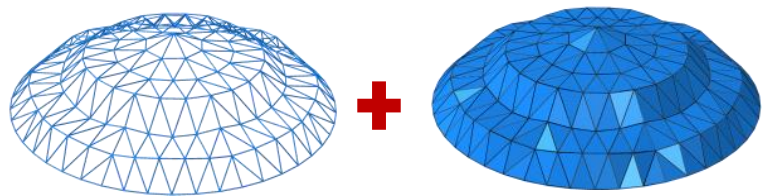

(a) The 1 st-order buckling mode (shell)+the 1 st-order buckling mode (roofing system) (BB)

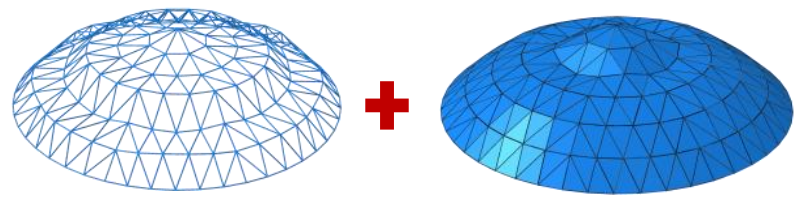

(b) The 1st-order buckling mode (shell)+the consistent imperfection mode (1) (roofing system) (B1)

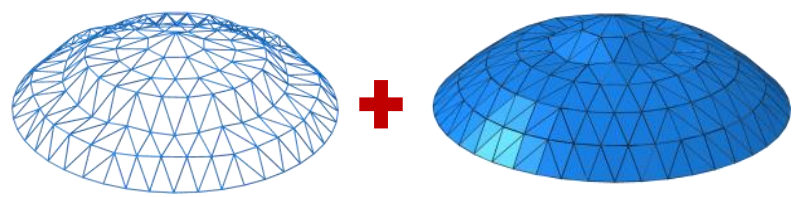

(c) The 1 st-order buckling mode (shell)+the consistent imperfection mode (2) (roofing system) (B2)

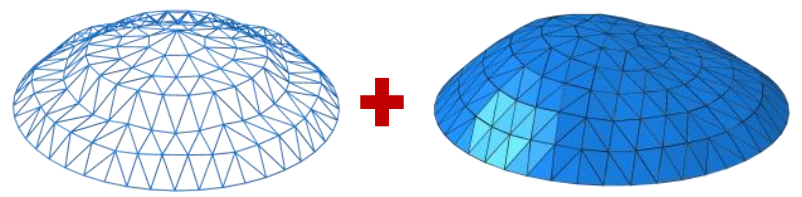

(d) The 1 st-order buckling mode (shell)+the 1 st-order frequency mode (roofing system) (BF)

Fig. 11 The combination of different initial imperfection modes (L/30)

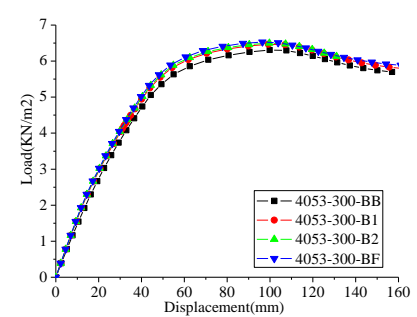

(a) Bolted purlin roofing systems

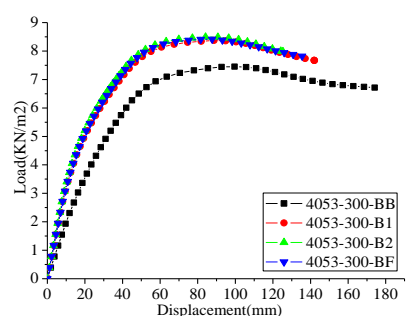

(b) Welded purlin roofing systems
Fig. 12 Load-displacement curves of shells under different combinations of the initial

imperfection modes

The above analysis were obtained for the case in which the initial imperfection modes for the reticulated shell structure and roofing system are equivalent. Nevertheless, further analyses are required for the case in which the imperfection modes of the shell and roofing system are different. Therefore, four kinds of initial imperfection modes are analysed in this paper, as indicated in Table 1. The 1st-order buckling mode of the reticulated shells is used as the initial imperfection modes of the reticulated shells, and the initial imperfections for the roofing system are set as follows: 1st-order buckling mode, consistent imperfection mode (1), consistent imperfection mode (2) and the 1st order frequency mode of the reticulated shell, as shown in Fig. 11. In consistent imperfection mode (1), the calculations are performed using the Riks method in ABAQUS, the differences in the displacement of the vertices in the reticulated shell between the step ((i-1)th step) before the peak value of the static bearing capacity occurs (ith step) and the step ((i+1)th) after the peak value are estimated. In consistent imperfection mode (2), the 
calculations are performed using the General method in ABAQUS; specifically, the differences in the displacement of the vertices in the reticulated shell between the (i-1)th step before the peak value and the instant of the peak value (ith step) are estimated.

Fig. 12 shows that the influence of the different initial imperfection modes on the shell bearing capacity is smaller in the case of the bolted purlin roofing system (fluctuations within $3.5 \%$ ) than in the case of the welded purlin roofing system (13.6\%). When the imperfection modes of the reticulated shell and roofing system are equivalent, the adverse effects of the initial imperfections are more notable.

\subsection{Influence of the purlin joint}

According to the analysis in Section 3, the rigidity and strength of the purlin joints are key factors influencing the increase in the structural bearing capacity owing to the roofing system, and their role should be further analysed.

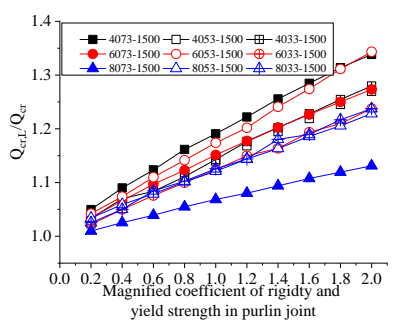
system-L/1500

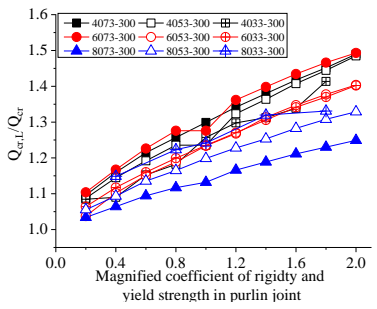

(c)Reticulated shells with welded roofing system-L/300
(a)Reticulated shells with welded roofing

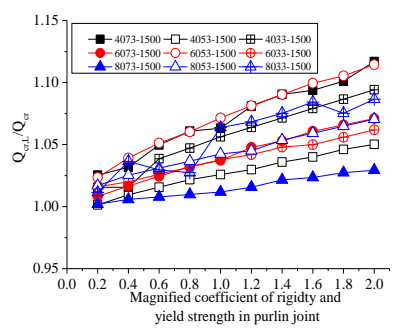

(b)Reticulated shells with bolted roofing system-L/1500

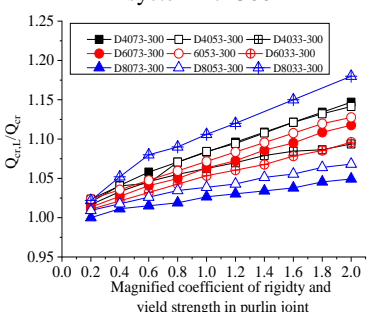

(d)Reticulated shells with bolted roofing system-L/300
Fig. 13 Ratio of peak load to magnified coefficient of rigidity and yield strength in joints

Fig. 13 shows that the static bearing capacity of the reticulated shell increases with an increase in the axial rigidity, rotational rigidity and yield strength in the purlin joints. As the purlin joint rigidity and yield strength increase from 0.2 to 2.0 times those of the original purlin joint, the effect of the welded and bolted purlin roofing systems on the bearing capacity of the reticulated shells increases by $1-34 \%$ and $1-11.7 \%$ under the initial imperfection of $\mathrm{L} / 1500$, respectively. Under the initial imperfection of $\mathrm{L} / .300$, the influence of the welded and bolted purlin roofing systems on the bearing capacity increases from $3.4 \%$ to $49.3 \%$ and $0 \%$ to $18 \%$, respectively. When the rise span ratio of the reticulated shell is $1 / 7$ and the span is $80 \mathrm{~m}$, the amplitude of the bearing capacity increases as the rigidity and yield strength of the purlin joints are lower than those under the other conditions, and the amplitude tends to decrease as the span increases. A similar phenomenon can be observed in the shell with a rise span ratio and initial imperfection of $1 / 5$ and $\mathrm{L} / 300$, respectively.

\subsection{Influence of the pillar height}

Pillars are critical load-bearing members and assist the roofing system in improving the static stability of the shells. The influences of the pillar height $(0.2 \mathrm{~m}, 0.6 \mathrm{~m}$ and $1.0 \mathrm{~m})$ on the static stability of reticulated shells with different spans and rise span ratios are analysed. The initial imperfection amplitude of the reticulated shell structure is $L / 300$.

As shown in Fig. 14, the pillar height significantly influences the static stability of the shell structures. With an increase in the pillar height, the static bearing capacity and structural rigidity of the shell increase significantly. When the pillar height is $0.2 \mathrm{~m}$ in the D8073-300 shell, the static bearing capacity of the shell with the bolted purlin and welded roofing systems increases by $3.2 \%$ and $15.6 \%$, respectively. When the pillar height is $1.0 \mathrm{~m}$, the static bearing capacity of the shell of the bolted and welded roofing systems increases by $20.3 \%$ and $60 \%$, respectively. The rigidity of the vertex with the largest displacement in the reticulated shell is 1.26 and 1.65 times higher when the pillar height is $1.0 \mathrm{~m}$ than that when the height is $0.2 \mathrm{~m}$. Similar phenomena can also be observed in the shells with other spans and rise span ratios.

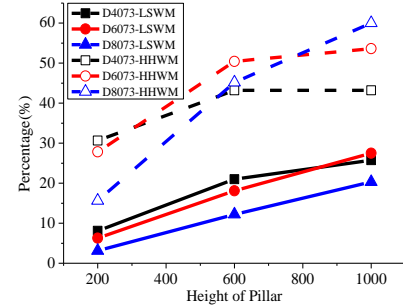

(a) Influence of the pillar height on the bearing capacity of the shells with different spans

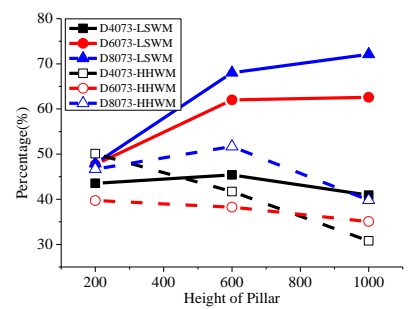

(c) Influence of the pillar height on the axial yield ratio of the purlin joints of shells with different spans

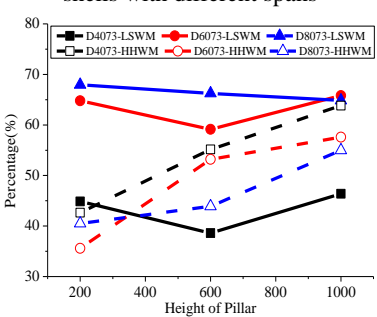

(e) Influence of the pillar height on the bending moment yield ratio of the joints of shells with different spans

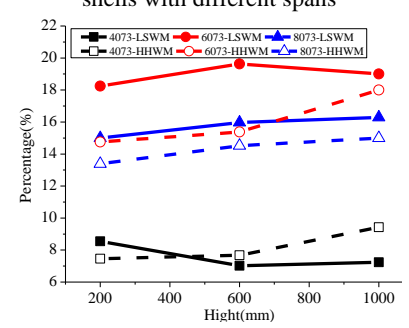

(g) 1P percentages in the shells with different pillar heights and spans

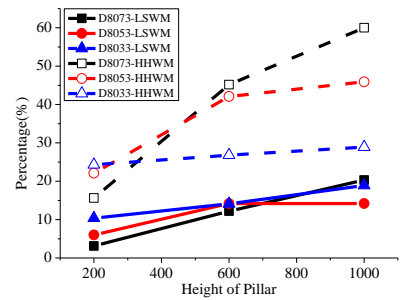

(b) Influence of the pillar height on the bearing capacity of the shells with different rise span ratios

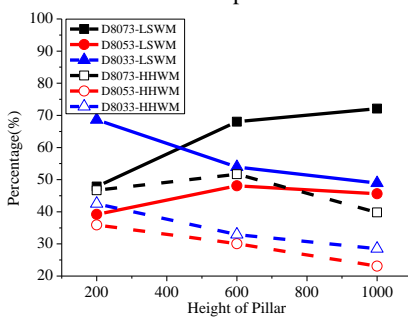

(d) Influence of the pillar height on the axial yield ratio of the purlin joints of shells with different rise span ratios

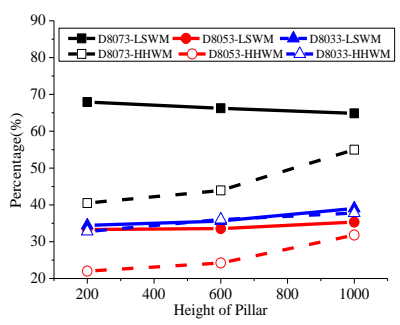

(f) Influence of the pillar height on the bending moment yield ratio of the joints of shells with different rise span ratios

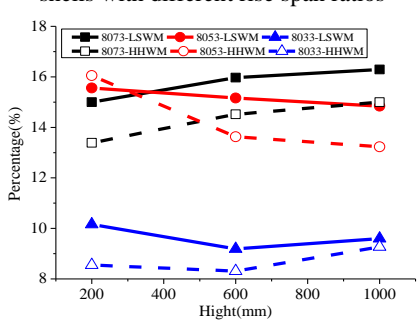

(h) $1 \mathrm{P}$ percentages in the shell with different pillar heights and rise span ratios
Fig. 14 Influence of the pillar height on the bearing capacity, yield ratio of purlin joints

$$
\text { and } 1 \mathrm{P} \text { percentages of the shell members }
$$

When the rise span ratio is $1 / 7$, at the peak value of the static bearing capacity of the shell with the welded purlin roofing system, the proportion of the 1P plastic shell members increases as the pillar height increases, this trend is not significant in the case of the shell with the bolted purlin roofing system. Due to the weak constraint of the bolted purlin joints when the reticulated shell bears a larger static load, the bolted purlin joints aggravate the local instability more notably, and the proportion of the plastic shell members decreases slightly. The cross-sections of the shell members, purlins and roof panels are similar to the composite section beams, considering the fully transmitted load for the purlins joints, as the distance between the section centroids of the purlin and shell members increases, the rigidity and bearing capacity of the reticulated shell structure is significantly improved. Figs. 14 (e) and (f) show that when the purlin joints have a welded connection, the axial constraints of the welded joints are sufficiently strong to transmit the axial load, and the yield proportion of the out-of-surface bending moment constraint in the purlin joints increases as the pillar height increases. When the purlin joints are bolted connections, with weaker axial constraints, more purlin joints enter the plastic stage earlier, and the pillar height does not notably influence the yield proportion of the bolt purlin joints. 
According to above analysis results, when the pillar height is small, the rigidity of the reticulated shell membrane can be increased by the roofing system, and the increase in the purlin section and axial constraint of the purlin joints can enhance the increase in the shell static stability owing to the roofing system. When the pillar height is large, the purlins bear a larger bending moment, and the purlin joints need provide sufficient bending moment constraints for the influence of the roofing system to be notable.

\subsection{Influence of the roof load}

The effect of the roof load on the static stability of the reticulated shells with different spans, rise span ratios and imperfection amplitudes is analysed.

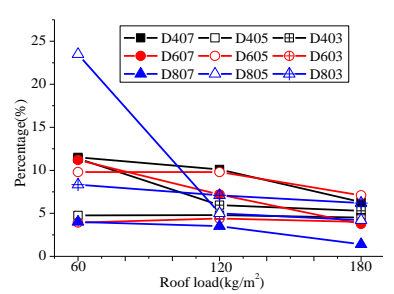

(a) Bolted purlin roofing system with an L/1500 imperfection

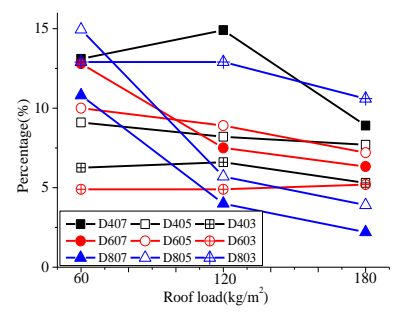

(c) Bolted purlin roofing system with an L/300 imperfection

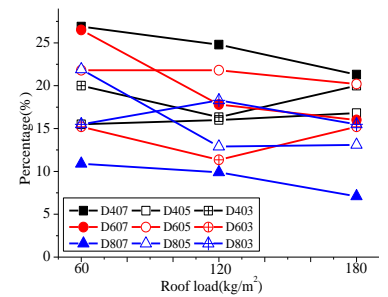

(b) Welded purlin roofing system with an L/1500 imperfection

(d) Welded purlin roofing system with an L/300 imperfection

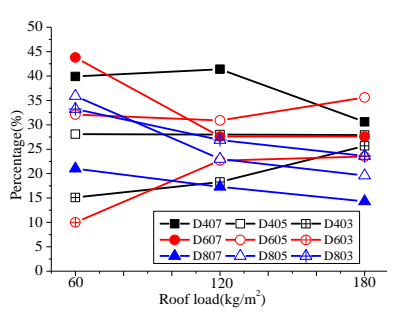

Fig. 15 Effect of the roof loads on the shell bearing capacity

Fig. 15 shows that the effect of the bolted purlin roofing system on the static bearing capacity of the shell decreases with increasing roof load. The bolted purlin roofing system does not notably influence the static bearing capacity of a shell with a large span, small rise span ratio and large roof load; nevertheless, its effect is notable in the case of a shell with a small span, large rise span ratio and a lighter roof load. When the rise span ratios are 1/5 and $1 / 7$, the effect of the welded purlin roofing system on the shell bearing capacity decreases with an increasing roof load. When the shell span is $80 \mathrm{~m}$ and the roof load is larger $(120 \mathrm{~kg} / \mathrm{m} 2$ and $180 \mathrm{~kg} / \mathrm{m} 2)$, the influence of the roofing system on the shell increases as the rise span ratio of the shell increases.

4.6. Statistical results of the effect of the roofing system on the static bearing capacity

The statistical analysis of the effect of the roofing systems on the static bearing capacity of the reticulated shell structures with different roof loads, spans, rise span ratios and imperfection amplitudes indicates that the influence is in accordance with the normal distribution, with the coefficient of confidence being 95\%, as shown in Fig. 16. The effect of the roofing systems on the static bearing capacity and the effect of the skin of the roof panels on the bearing capacity of a shell with only purlin systems are also indicated in Table 2 .

Table 1

Statistics of the effect of the roofing systems on the static bearing capacity of the reticulated shell

\begin{tabular}{|c|c|c|c|c|c|c|c|}
\hline \multirow{2}{*}{ Roofing system } & \multirow{2}{*}{$\begin{array}{c}\text { Initial imperfection } \\
\text { amplitude }\end{array}$} & \multicolumn{4}{|c|}{ Effect of the roofing system $(\%)$} & \multicolumn{2}{|c|}{ Effect of the roof panels (\%) } \\
\hline & & Expected value & Standard deviation & Maximum & Minimum & Maximum & Minimum \\
\hline \multirow{2}{*}{ Bolted purlin roofing system } & $\mathrm{L} / 1500$ & 7.00 & 4.28 & 23.50 & 1.40 & 3.13 & 0.39 \\
\hline & $\mathrm{L} / 300$ & 8.36 & 3.50 & 14.94 & 2.20 & 5.22 & 0.26 \\
\hline \multirow{2}{*}{ Welded purlin roofing system } & $\mathrm{L} / 1500$ & 17.36 & 4.92 & 26.90 & 7.10 & 6.6 & 0.37 \\
\hline & $\mathrm{L} / 300$ & 26.80 & 8.36 & 43.80 & 10.00 & 7.10 & 0.27 \\
\hline
\end{tabular}

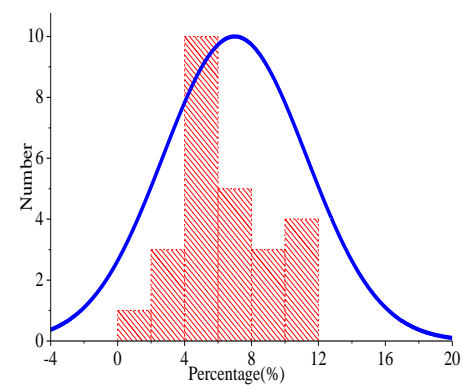

(a) Bolted purlin roofing systems influence on the shell with L/1500 imperfection

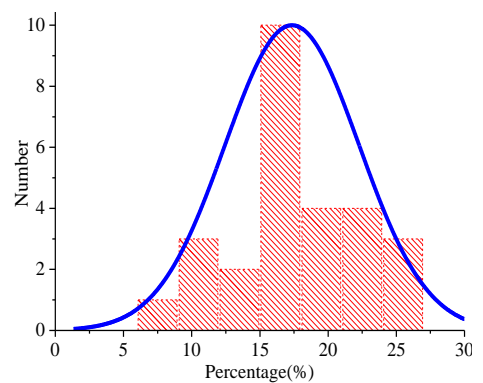

(b) Welded purlin roofing systems influence on the shell with L/1500 imperfection

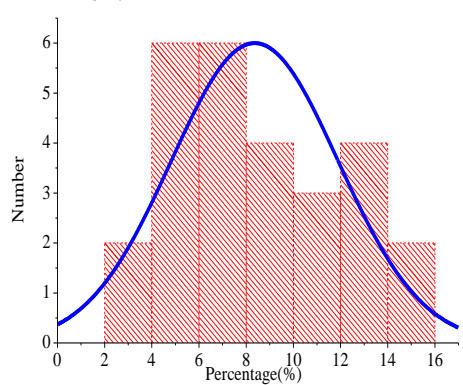

(c) Bolted purlin roofing systems influence on the shell with L/300 imperfection

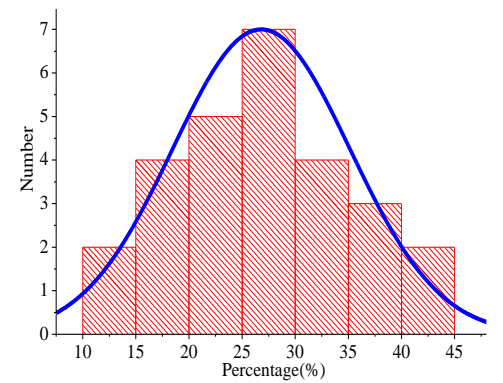

(d) Welded purlin roofing systems influence on the shell with L/300 imperfection

Fig. 16 Histogram of the effect of the roofing systems on the bearing capacity of the reticulated shell 


\section{Conclusion}

The FEAs of the reticulated shell structures with different roofing systems were performed based on experimental analyses, and the results indicated that the roofing systems notably influence the static stability of reticulated shell structures. The following conclusions were derived:

(1). Roofing systems can significantly increase the static bearing capacity of reticulated shell structures and intensify the shell members' plastic development around the location of the initial imperfection at the peak bearing capacity. The influence of the roofing systems should be considered in the design and analysis of the reticulated shell structures, in terms of the static stability, the roofing systems notably affect the nonlinear whole-process failure mode of the reticulated shell structures under a static load.

(2). The roofing system considerably affects the static bearing capacity of different reticulated shell structures and exhibits certain characteristics. The influence of the roofing systems on the bearing capacity of a shell with an 80 m span (roof load of $120 \mathrm{~kg} / \mathrm{m} 2$ or $180 \mathrm{~kg} / \mathrm{m} 2$ ) increases with an increase in the rise span ratios. Furthermore, this influence increases with an increase in the imperfection amplitude, and the trends related to the welded purlin roofing systems are more notable. When the initial imperfection modes of the roofing systems and reticulated shell structures are equivalent, the initial imperfection is detrimental to the shell bearing capacity.

(3). In roofing systems, the purlin systems are the key factors to increase the static bearing capacity of the reticulated shell structures, and the purlin joints help intensify the effect of the purlin systems on the reticulated shell structures. In most cases, even when the shell is subjected to the highest static load, no plastic deformation occurs in any of the roofing system components. The axial constraints and out-of-surface bending moment constraints of the purlin joints are critical factors. In the design and analysis of reticulated shell structures, when considering the effect of the roofing system on the bearing capacity of reticulated shells, sufficient strength and rigidity of the purlin joints must be ensured during construction.

(4). The influences of the roofing systems' components on the reticulated shell structures' static stability are analysed. The pillars are important force transmission components, and the roofing systems' influences on reticulated shells' static bearing capacity increase with increasing pillar height. The roofing system's influences on the reticulated shell structures' static bearing capacity increases with increasing axial rigidity, rotational rigidity and yield strength in the purlin joints, the trend is more significant in the welded purlin roofing system. The roof panels' skin effects on the reticulated shells structures' static bearing capacity with L/1500 initial imperfection are $0.39 \%-3.13 \%$ and $0.37 \%-6.6 \%$ for shells with bolted and welded purlin systems respectively. The roof panels' skin effects on the reticulated shell structures' static bearing capacity with $L / 300$ initial imperfection are $0.26 \%-5.22 \%$ and $0.27 \%-7.1 \%$ for shells with bolted and welded purlin systems respectively.

(5). The effect of the roofing systems on the bearing capacity of the reticulated shell structures with different factors is in agreement with the normal distribution, and the numerical values of these characteristics are listed in Table 2. The results can provide a reference for the design and analysis of reticulated shell structures.

\section{Acknowledgements}

This study was supported by the National Key Research and Development Program of China (2018YFC1504304), the National Nature Science Foundation of China (No. 51778183) and the National Science Foundation for Distinguished Young Scholars of China (No. 51525802). The authors extend their sincere gratitude for the support.

\section{References}

[1] Shizhao S. and Xi C., Stability of Reticulated Shells, Science Press, Beijing, 1999.

[2] Fan F, Cao Z.G., and Shen S.Z., "Elasto-plastic stability of single-layer reticulated shells", Thin Walled Struct, 48, 827-836, 2010.

[3] Feng F, Zhenggang C, Huihuan M, and Jiachuan Y., Elasto-plastic Stability of Reticulated Shells. Science Press, Beijing, 2015.

[4] Zhi X.D., Fan F., and Shen S.Z., "Elasto-plastic instability of single-layer reticulated shells under dynamic actions", Thin Walled Struct, 48, 837-845, 2010.

[5] Zhi X.D., Fan F., and Shen S.Z., "Failure of the single layer reticular dome under earthquake", China Civil Engineering Journal, 38, 11-20, 2005.

[6] M. Fong, Y.P. Liu, and S.L. Chan, "Second-Order Analysis and Experiments of Semi-Rigid and Imperfect Domes", Advances in Structural Engineering, 15(9), 1537-1546, 2012.

[7] S.Kato, I. Mutoh and M. Shomura, "Effect of joint rigidity on buckling strength of single layer lattice domes", Bulletin of the International Association for Shell and Spatial Structures, Madrid, Spain, 35, 101-109, 1994.
[8] Kato S., Mutoh I. and Shomura M., "Collapse of semi-rigidly jointed reticulated domes with initial geometric imperfections", J Construct Steel Res, 48, 145-68, 1998.

[9] Huihuan M., Feng F., Peng W., Zhang H. and Shen S.Z., "Experimental and numerical studies on a single-layer cylindrical reticulated shell with semi-rigid joints", Thin Walled Struct, 86, 1-9, 2015

[10] Ma Huihuan, Ma Yueyang, Yu Zhiwei and Fan Feng, "Experimental and numerical research on gear-bolt joint for free-form grid spatial structures", Engineering Structures, 148, 522-540, 2017.

[11] Si Wei Liu, Rui Bai, Siu-Lai Chan and Yao-Peng Liu, "Second-Order Direct Analysis of Dome-like Structures Consisting of Tapered Members with I-Sections", Journal of Structural Engineering, 142(5), 04016009-1-11, 2016.

[12] Rui Bai, Si-Wei Liu and Siu-Lai Chan, "Modal and Elastic Time-History Analysis of Frames with Tapered Sections by Non-Prismatic Elements", International Journal of Structural Stability and Dynamics, 18(9), 1850106-1-34, 2018.

[13] Yao-Peng Liu, Siu-Lai Chan, Zuo-Lei Du and Jian-Wei He, "Second-order direct analysis of long-span roof structures", EUROSTEEL, 3930-3939, 2017.

[14] Y. P. Liu, S. J. Pan, Simon W. K. Leung and S. L. Chan, "Design and construction of long-span single-layer dome structures by direct analysis", HKIE TRANSACTIONS, 25(1), 29-43, 2018.

[15] Xie Xiangyang, Chen Guo and Yin Lei, "Key Technology in Design and Construction of Single-layer Reticulated Shell of H-section Units in MGM resorts Development LCC, Macau", Construction Technology, 45(21), 61-65, 2016.

[16] Code for design of steel structures (GB50017-2017), China Planning Press, China, 2017.

[17] Eurocode 3 Design of steel structures Part 1-6: Strength and Stability of Shell Structures, European Committee For Standardization, the European Union Per Regulation, 2007.

[18] Eurocode 3 Design of steel structures Part 1-3: General rules-Supplementary rules for cold-formed members and sheeting, European Committee For Standardization, the European Union Per Regulation, 2011.

[19] Technical Specification for Space Frame Structures (JGJ 7-2010), China Architecture \& Building Press, China, 2010

[20] Yongjun He and Xuhong Zhou, "Research on load bearing of the reinforced concrete roof board cooperative with grid structure", J Harbin Inst Technol, 39, 188-91, 2007.

[21] Yizhe Z., "Shaking table test and performance study of single-layer reticulated cylindrical shell with skin diaphragm", Beijing university of technology, Beijing, 2012.

[22] Maria Koliou and Andre Filiatrault, "Buildings with Rigid Walls and Flexible Roof Diaphragms. I: Evaluation of Current U.S. Seismic Provisions", Journal of Structural Engineering, 142(3), 04015166, 1-13, 2016

[23] Maria Koliou and Andre Filiatrault, "Buildings with Rigid Walls and Flexible Roof Diaphragms. II: Evaluation of a New Seismic Design Approach Based on Distributed Diaphragm Yielding. Journal of Structural Engineering", 142(3), 04015167, 1-9, 2016.

[24] Li Wenliang, Zhi Xudong, Wang Duozhi, Fan Feng and Shen Shizhao, "Static stability analysis of a reticulated shell with a roofing system", Engineering Structures, 185, 315-331, 2019.

[25] Xudong Z., Zhang T., Wenliang L. and Feng F., "Effect of roofing system on elasto-plastic stability and seismic performance of single-layer reticulated shells", China Civil Engineering Journal, 50, 19-26, 2017.

[26] Duozhi W., Wenliang L., and Xudong Z., "Static stability analysis of reticulated shell with purlin roofing system", Eng Mech, 34 (Suppl), 71-77, 2017.

[27] Xie Xiangyang, Chen Guo and Yin Lei, "Multi-parameter simulation method of semi-rigid node of steel tubular scaffold with coulpers", Journal of Civil and Environmental Engineering, 41(4), 92-103, 2019. 